\title{
Active alignment for two-beam interferometers
}

\author{
Dimitrios Kalamatianos ${ }^{\mathrm{a})}$ and Peter E. Wellstead \\ Hamilton Institute, National University of Ireland, Maynooth, Ireland \\ John M. Edmunds \\ Acimetrics Ltd., Manchester Science Park, Lloyd St. North, Manchester M15 6JJ, United Kingdom \\ Panos Liatsis \\ School of Engineering and Mathematical Sciences, City University, Northampton Square, London EC1V OHB, \\ United Kingdom
}

(Received 21 July 2005; accepted 16 November 2005; published online 25 January 2006)

\begin{abstract}
An active control system is described for the automatic alignment of the mirrors in a two-beam interferometer. From an initial unaligned position the active control system determines the degree of misalignment and adjusts the relative mirror positions accordingly. An embodiment of the system is described for a Michelson interferometer in which one of the mirrors is mounted upon three piezoelectric transducers (PZT) arranged so that they can both tilt and retard the mirror. Laser sources and corresponding photodetectors are also incorporated such that a control system can use the PZT actuators to produce a series of mirror movements relative to the fixed mirror and give a set of two-dimensional diffraction patterns - one for each of the laser photodetectors. Amplitude and phase information is extracted from these patterns which enables the control system to align the mirrors such that the diffraction pattern maxima is at the center of the instrument central photodetector. In a further stage of the control algorithm, the alignment accuracy is refined using information from the laser photodetectors during retardation of the mirror. In this manner, the initial mirror alignment, maintaining that alignment during retardation and diagnosis of possible misalignment, become part of a single active control technology for instrument alignment. (C) 2006 American Institute of Physics. [DOI: 10.1063/1.2150823]
\end{abstract}

\section{INTRODUCTION}

Two-beam interferometers, as exemplified by the Michelson instrument, are basic to Fourier transform near-infrared (FT-NIR) spectroscopic measurement. ${ }^{1}$ However, the quality and validity of measurements from such instruments is dependent upon the precise relative alignment of the optical components, and maintaining that alignment during operation, or at least the rapid diagnosis of misalignment. Thus, the task of aligning the optical components of a Michelson interferometer and maintaining that alignment is a subject of concern to instrument designers and end users. The traditional approach to FT-NIR interferometer design is to make the instrument hardware physically robust, and to use antivibration mountings, such that after initial setup the optics remain in alignment. This approach is suitable for FT-NIR interferometry conducted off-line in the controlled environment of an analytical laboratory where the instrument's physical size is not important. However, there is a growing trend toward NIR spectroscopic measurement at-line or online, and for these applications there is a need for physically compact FT-NIR instruments that incorporate active control systems which automatically align and retain the alignment of the optics in difficult environmental conditions.

While our interest is in at-line and on-line instruments, the algorithm described here is generally applicable in two-

\footnotetext{
a)Electronic mail: dimitris.kalamatianos@nuim.ie
}

beam interferometry. Specifically, we describe how active control methods can be used to automate the initial alignment of the mirrors in a Michelson interferometer, and further how active control can maintain alignment during use, e.g., after a large environmental shock, ${ }^{2}$ and provide diagnostic information on measurement quality. The principal benefit of active control on optical alignment is that interferometers based on this principle can be physically compact and, hence, portable. An additional advantage is that the instrument control system can immediately diagnose alignment problems, automatically realign and report on potentially suspect measurements. The implications of this are that with active control and alignment FT-NIR spectroscopy can be used on-line and in many more challenging circumstances than the traditional analytical laboratory.

We now describe the basic issues of mirror alignment and the associated accuracy requirements. Although a solidly designed beam-splitter housing combined with a finely machined mirror carriage can reduce the amount of misalignment, the mirror displacement system, in common with other dynamic systems, will exhibit a certain amount of mechanical compliance. Consequently, the moving mirror tends to tilt slightly or wobble as it is displaced and, as such, will not be precisely perpendicular to the incident beam at all times. The net result of this phenomenon is that as the beams from each arm of the interferometer are recombined, the resultant beam is displaced from the optical axis, as shown in Fig. 1. Since the angle of tilt is not constant, the central fringe does not 


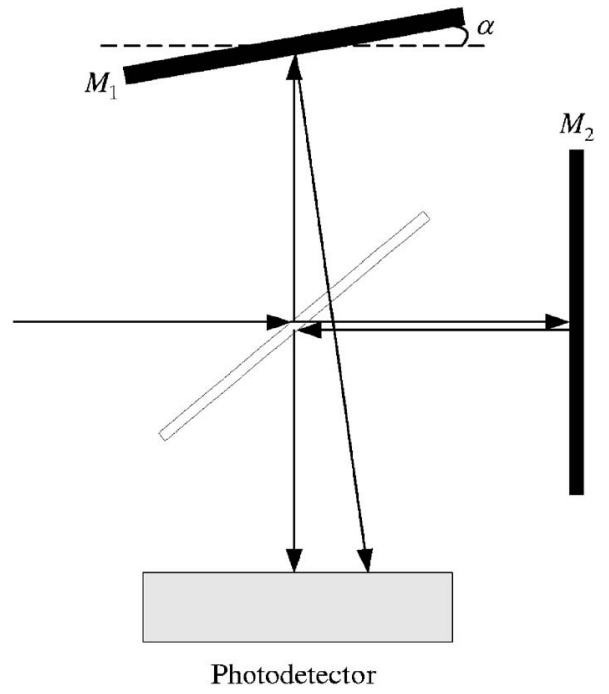

FIG. 1. Schematic diagram of the Michelson's optics showing how tilting the moving mirror $M_{1}$ causes the recombinant beams to diverge from the optical axis, thus causing a shift to the fringe pattern.

remain in a fixed position, but tends to drift about the optical axis. Thus, the central fringe and the photodetector become misaligned with the effect that the interferogram is not obtained from the true center of the fringe pattern. Hence, from one scan to the next, the interferogram's magnitude or envelope changes as the visibility or modulation index of the fringes varies.

In Fig. 1, the angle of tilt, $\alpha$, is greatly exaggerated. In reality, the maximum value is of the order of $100 \mu \mathrm{rad}^{3}$ However, even a tilt angle of this magnitude is sufficient to cause a significant shift to the position of the diffraction fringes and to prejudice the quality of the detected signal. In order to further quantify the effect of mirror tilt on the spectrum, we elaborate upon the calculations of Williams ${ }^{3}$ by considering the ideal interferogram defined by the cosine transform of the spectrum given by

$$
I(\delta)=\int_{-\infty}^{\infty} B(\sigma) \cos (2 \pi \sigma \delta) d \sigma,
$$

where $\delta$ is the optical path difference and $\sigma$ is the wave number. According to Williams, ${ }^{3}$ the attenuation to the distorted interferogram $I\left(\delta^{\prime}\right)$ can be modeled by the bracketed term in Eq. (2):

$$
I\left(\delta^{\prime}\right)=\int_{-\infty}^{\infty} B(\sigma)\left[\frac{2 J_{1}(4 \pi \sigma r \alpha)}{4 \pi \sigma r \alpha}\right] \cos (2 \pi \sigma \delta) d \sigma,
$$

where $r$ is the radius of the beam through the instrument, $\alpha$ is the angle of tilt and $J_{1}(\cdot)$ is an order one Bessel function of the first kind, which can be approximated for arguments less than $\approx 2$ by

$$
J_{1}(x)=\frac{x}{2}\left(1-\frac{x^{2}}{8}\right) .
$$

According to Cohen, ${ }^{4}$ the attenuation term in Eq. (2) can be approximated by

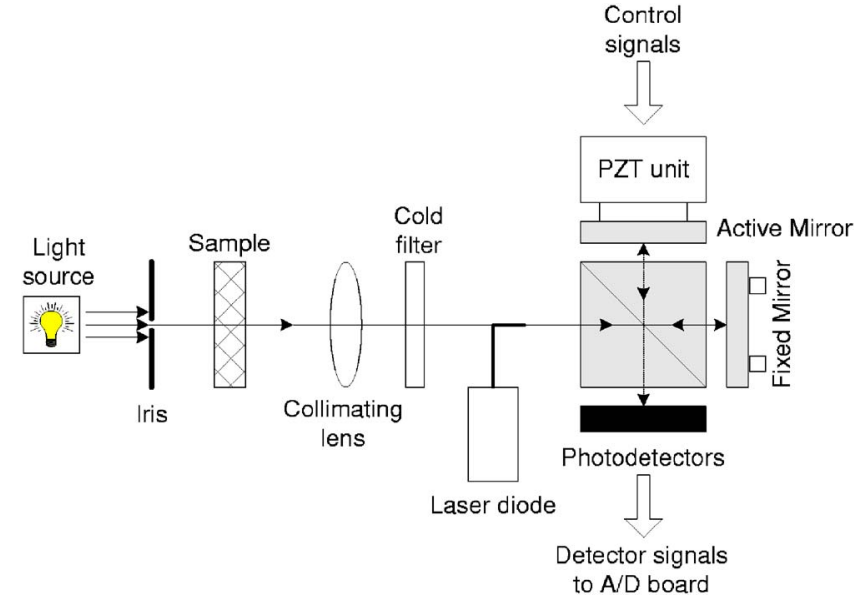

FIG. 2. (Color online) Schematic diagram of the FT-NIR spectrometer in transmission mode. White light passes through the sample and into the Michelson for analysis. Interference fringes from the laser diode are used for control.

$$
\frac{2 J_{1}(4 \pi \sigma r \alpha)}{4 \pi \sigma r \alpha} \approx 1-A \sigma^{2} \alpha^{2},
$$

where $A=2 \pi^{2} r^{2}$

It can be seen that, for a given wave number $\sigma$ and fixed $r$, the value of the Bessel function is dependent on the size of $\alpha$. As the value of the bracketed term in Eq. (2) approaches zero, the magnitude of the interferogram tends towards zero. Conversely, as this term approaches unity, the magnitude of the interferogram tends to its maximum value of unity where perfect alignment is assumed.

In fact, Williams quotes figures for these two conditions of $4 \pi r \sigma \alpha=3.83$ and 0.283 , respectively. From an operational viewpoint, if a $5 \%$ variation from the maximum is tolerable, then, from Bessel tables, ${ }^{5}$ the value of $4 \pi r \sigma \alpha$ must not exceed 0.6 . For a $2 \%$ variation, this figure is reduced to 0.4 . Therefore, given the value of the term in parenthesis for a particular wave number, it is possible to determine the maximum angle of tilt that is acceptable. For example, consider a monochromatic source such as a laser diode with a wavelength of $670 \mathrm{~nm}$, i.e., $\sigma \approx 15000 \mathrm{~cm}^{-1}$, and a beam radius $r=1.25 \mathrm{~cm}$. The value of $\alpha$ is calculated to be approximately equal to $1.7 \mathrm{mrad}$ for a $2 \%$ variation and $2.7 \mathrm{mrad}$ for a $5 \%$ variation. An important thing to note from Eq. (2) is that the bandwidth of the spectrum is not affected by the tilting of the mirror. However, the shape of the envelope is altered by the attenuation of each of the spectrum's component wavelengths. The earlier calculations are of course theoretical, and in practice would be further conditioned by the geometry of instrument detector and other aspects of the instrument function. ${ }^{6}$

\section{EXPERIMENTAL SYSTEM}

In this section we describe key features of an experimental Michelson interferometer used to demonstrate the principles of automatic alignment. A schematic diagram of the experimental system in transmission mode is shown in Fig. 2. The framework of the instrument was constructed from a series of components manufactured by Spindler Hoyer under 
the trade name Microbench. The optical path through the interferometer begins at the collimating lens, which collects divergent radiation from the source and produces a parallel beam, as required by the system. Located directly behind the collimating lens, a "cold filter" was inserted to low-pass filter the source, i.e., to remove the visible portion of the source spectrum and pass only wavelengths at the near-infrared region and above. The source used was a quartz tungstenhalogen monofilament lamp, which has a useful operating range of $220 \mathrm{~nm}$ to $2700 \mathrm{~nm}$, i.e., from the ultraviolet through the visible band and into the infrared. ${ }^{7}$

At a distance of approximately $100 \mathrm{~mm}$ from the cold filter, a $20 \mathrm{~mm}$ fused silica corner cube beam-splitter assembly was located, by which the collimated source was amplitude divided into the two arms of the interferometer. The moving mirror was securely fixed onto a piezoactuator subsystem (Piezosystem Jena, model 1058) which has a maximum tilt of $2 \mathrm{mrad}$, maximum $z$ translation of approximately $\pm 25 \mu \mathrm{m}$ and an unloaded resonant frequency greater than $5 \mathrm{kHz}$. Note that this piezoelectric transducer has a rapid response time, much faster from that required in the active control system to be described in this article. More will be said of this in the next section.

The fixed mirror was mounted on a rigid kinematic mount, which facilitated manual alignment. Three adjusting screws gave three degrees of freedom to the fixed mirror's position that could then be locked into place via a central bolt. An approximate alignment was performed manually in such a manner after the initial construction. Both moving and fixed mirrors were manufactured from aluminium with a protective coating of silicon oxide. The mirrors having a diameter of $25 \mathrm{~mm}$ were optically flat to within $1 / 10$ of a wavelength at $514 \mathrm{~nm}$, according to the manufacturer's specifications (Optosigma).

Located close to the collimating lens and perpendicular to the optical path was a laser diode whose primary function was to provide the monochromatic fringes needed by the active alignment and alignment tracking algorithms. Monochromatic fringes were required for these two processes since they extend further than the fringes originating from the broadband source. In addition, the laser also provided an accurate method of calibrating mirror displacement, which in turn was required to determine calibration of subsequent spectra wavelength measurements. Note that during the retardation of the mirror a uniform time sampling method was used similar to that described by Brasunas and Cushman. ${ }^{8}$ In this scheme, the laser zero crossings provided a wavelength reference, while the user defined time samples allowed rapid sampling of the interferogram.

The laser diode had a wavelength of $670 \mathrm{~nm}$ and had a coherence length in excess of $10 \mathrm{~mm}$. In order to measure the amplitude of the laser fringes, four phototransistors, used as laser photodetectors, were mounted in the detector head and positioned equidistantly around the main NIR detector. The devices selected were Sharp PT510, which have a typical response of $600-900 \mathrm{~nm}$. The main photodetector selected was a Hamamatsu P203 8-03 lead selenium (PbSe) device having a sensitivity range of $1-5 \mu \mathrm{m}$, integral thermistor, and thermoelectric cooling plate. The interference

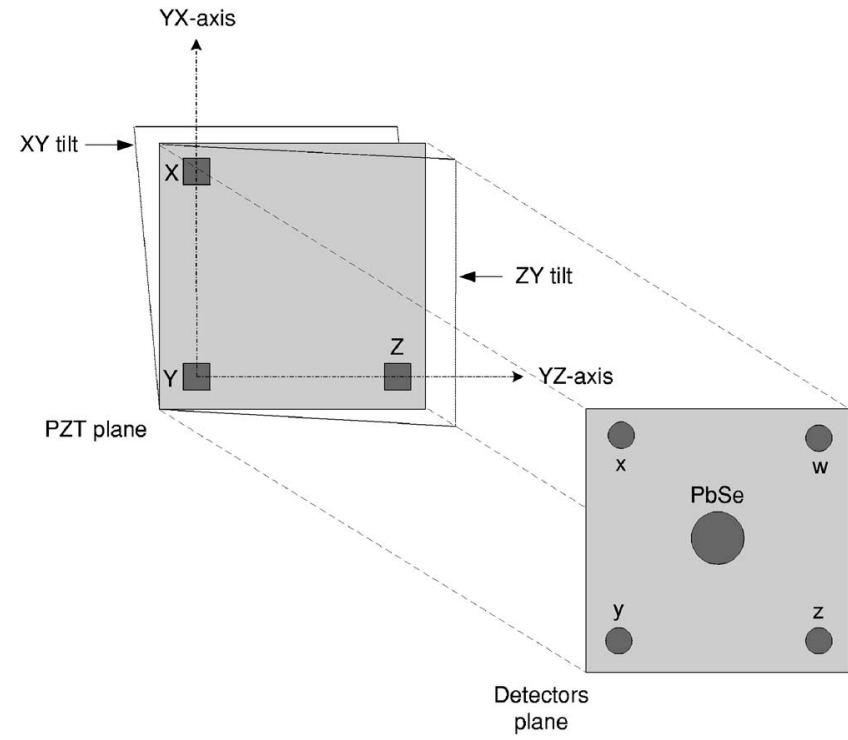

FIG. 3. Schematic diagram of PZT actuator layout and relative photodetector locations as viewed from the output plane.

fringes were imaged onto the photodetector by a fused silica meniscus lens having a focal length of $18 \mathrm{~mm}$ and a clear aperture of $10 \mathrm{~mm}$. This detector had an active area of $3 \mathrm{~mm}^{2}$ and a signal-to-noise ratio of approximately $32 \mathrm{~dB}$ at the intended operating voltage, i.e., $30 \mathrm{~V}$. An iris was placed between the light source and the interferometer, as shown in Fig. 2 to control the light levels within the instrument.

A schematic diagram of the piezoelectric transducers (PZT), $X, Y$, and $Z$, and the photodetectors, $x, y, z, w$, and $\mathrm{PbSe}$, as viewed from the output plane, i.e., the view looking into the instrument with the beam splitter removed, is shown in Fig. 3. The mirror represented by the gray square at the PZT plane is moved backwards and forwards by the means of control voltages applied to the PZT actuators attached to the rear of the mirror plate. Ideally, during the retardation process the actuators are extended by equal amounts, thus ensuring the parallel motion of the mirror perpendicular to the incident beam. This is ensured by either using matched PZTs, or making individual calibrations of each actuator.

Figure 3 also shows the relative positions of the phototransistors with respect to the PZTs. Four phototransistors are mounted on the detector head and positioned equidistantly around the NIR detector. By overlaying the photodetector head onto the PZT plane, it can be seen that phototransistors $z$ and $y$ lie on the $Y Z$ axis, while phototransistors $x$ and $y$ are coincidental with the $Y X$ axis. On successful completion of the self-alignment process, the mirror would be positioned at a point where the central fringe coincided with the PbSe photodetector. The PZT unit was used during this process to tilt the mirror and change the optical path difference at each phototransistor, as shown graphically in Fig. 3.

\section{ACTIVE ALIGNMENT}

\section{A. Description of the method}

Alignment is controlled in three distinct stages: a first stage which performs an approximate initial alignment based 


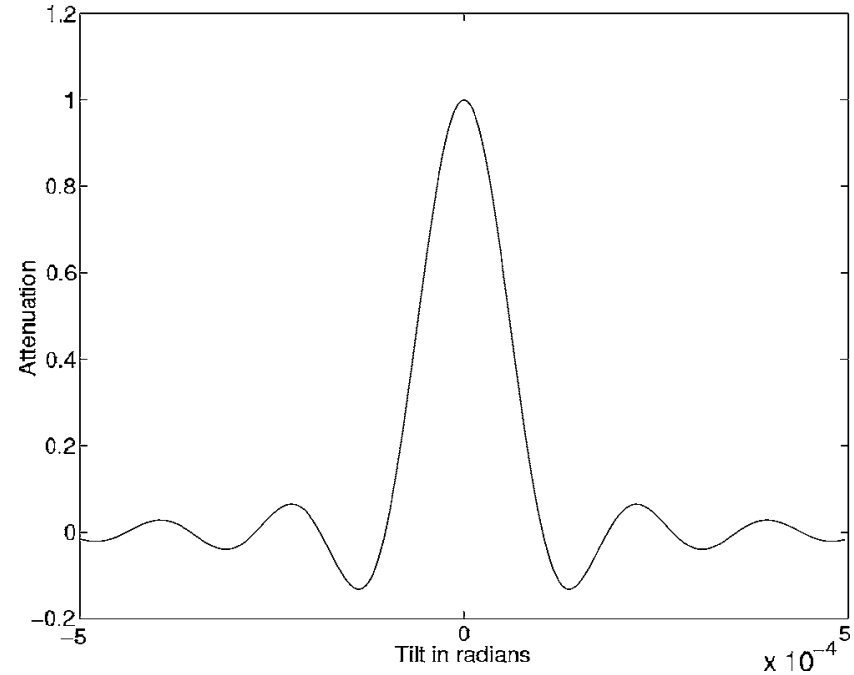

FIG. 4. Attenuation gain for mirror tilt in one axis.

on monitoring the amplitude of the outputs of the detectors, a second stage which relies upon the relative phase of beams reaching the detectors to provide an estimate of improved accuracy, and a third stage where the previous estimates are tested during normal operation of the instrument. The alignment process is described in detail later.

\section{Stage 1: Amplitude-based alignment}

When the mirrors in a Michelson interferometer are tilted and the light source is a monochromatic source (usually a laser) interference patterns are generated which are scanned across the laser phototransistors. The signals measured at the phototransistors depend on the optical path difference for the light from the two mirrors, and also on the misalignment between the mirrors. The interference pattern at each of the four phototransistors is determined by the optical path difference and the signal at each phototransistor is attenuated depending on the amount of misalignment. The attenuation also depends on the diameter of the phototransistor's detection area, with larger detectors areas being more sensitive to misalignments. The attenuation gain $K$ at a phototransistor signal can be approximated according to Eq. (4) by

$$
K \approx \frac{2 J_{1}(4 \pi \sigma r \alpha)}{4 \pi \sigma r \alpha},
$$

where $\sigma=1 / \lambda, \lambda$ is the source wavelength, $r$ is the detector radius, $J_{1}$ is an order one Bessel function of the first kind and $\alpha$ is the misalignment angle. Figure 4 shows the theoretical attenuation for a laser source of wavelength of $670 \mathrm{~nm}$, a detector of radius of $2 \mathrm{~mm}$ and the mirror tilt varying from $-5 \times 10^{-4} \mathrm{rad}$ to $5 \times 10^{-4} \mathrm{rad}$. This tilt is obtained with actuators $2 \mathrm{~cm}$ apart and moving $20 \mu \mathrm{m}$.

When the actuators are used to tilt the active mirror about axes $Y Z$ and $Y X$ the phototransistor signal at say $x\left(S_{x}\right)$ is given by the expression

$$
S_{x}=M+K p \cos (2 \pi \sigma \delta),
$$

where $M$ is the mean phototransistor signal, $K$ is the attenuation gain, $p$ is the peak modulation by the interferometer,

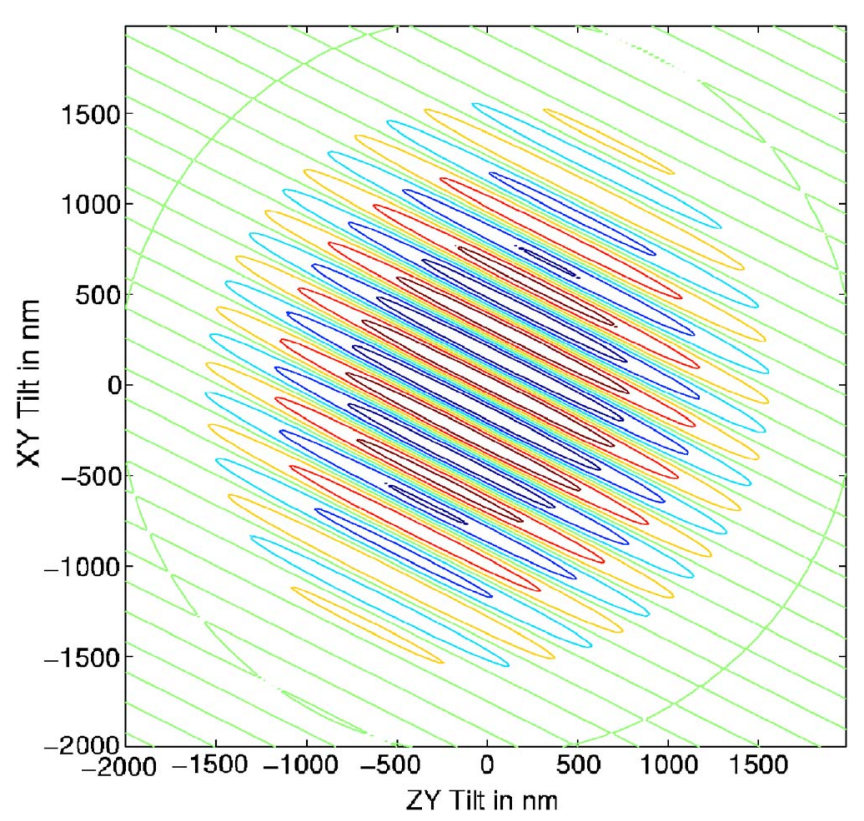

FIG. 5. (Color online) Contour of the expected phototransistor $x$ signal for different tilts and the $Y$ actuator constant.

and $\delta$ is the optical path difference for the light detected by the particular phototransistor.

Thus, if the $Y$ PZT is maintained stationary, Fig. 5 shows the contour of the expected amplitude variations in the detected phototransistor $x$ signal. This signal is also plotted in three-dimensional space in Fig. 6. The $Z Y$ tilt is the difference between the optical path difference at the $z$ phototransistor and that at the $y$ phototransistor. Likewise, the $X Y$ tilt is calculated by the difference between the optical path differences at $x$ and $y$ phototransistors. Figure 3 shows the $X Y$ and $Z Y$ tilts for the case that actuator $Y$ is kept constant.

The two-dimensional plot of Fig. 5 shows the contours of the phototransistor signal magnitude for the various tilts. The maximum phototransistor signal is at the center (corresponding to zero tilt in this idealized diagram, where it is assumed that the phototransistor is positioned in the center of the figure). It is possible to find the correct alignment point from the two-dimensional plot by finding the point at which

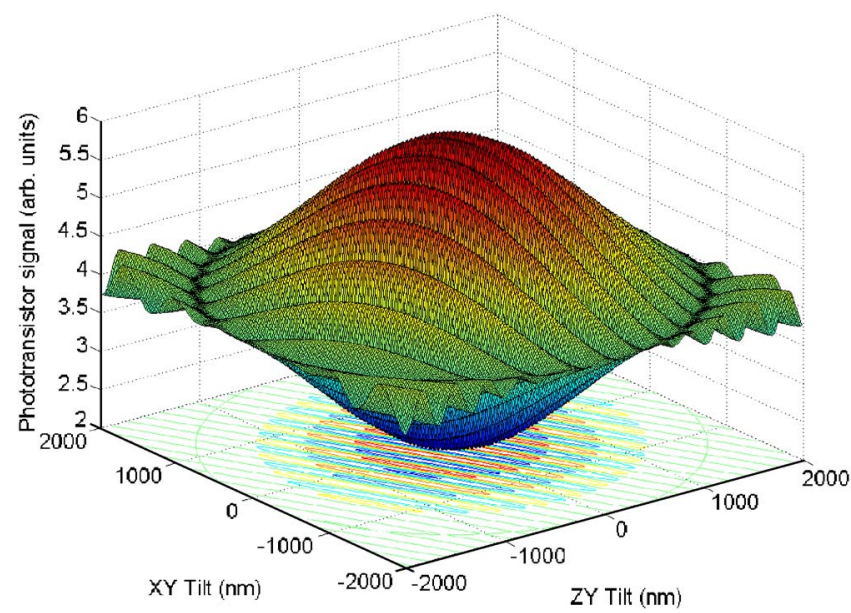

FIG. 6. (Color online) Three-dimensional surface plot of the expected phototransistor $x$ signal for different tilts and the $Y$ actuator constant. 

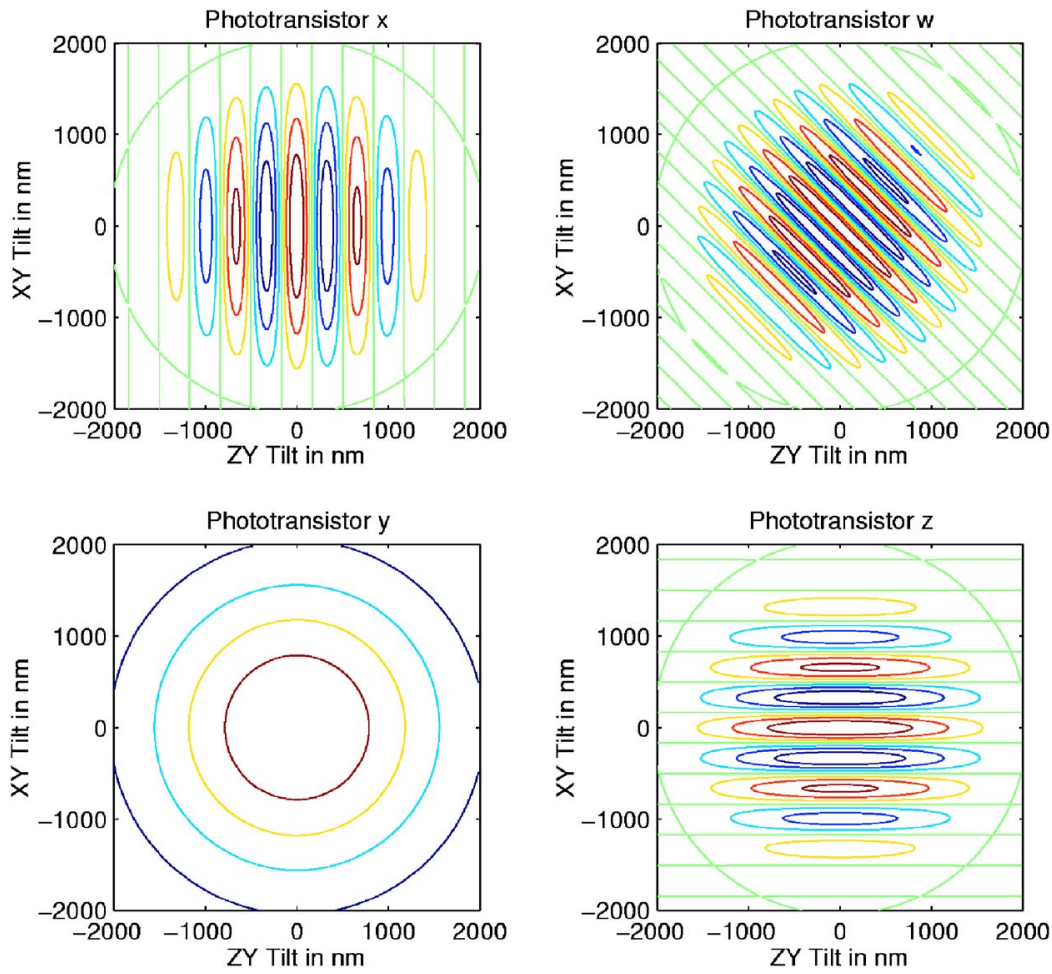

FIG. 7. (Color online) Contours of all the expected phototransistor signals for different tilts and the $Y$ actuator constant. the phototransistor signal is a maximum. Greater accuracy can be achieved by monitoring the amplitude at all four phototransistors. Figure 7 shows plots for all four phototransistors, assuming no movement of $Y$ actuator.

The signals from phototransistors $w, x$, and $z$ can be used to determine the mirror tilt position giving a maximum amplitude signal, but the signal from $y$ is not useful because the $Y$ actuator is not moving. To get a useful signal from all phototransistors, a ramp change is added to all the actuators at the same time as changing the $X Y$ and $Z Y$ actuator tilts. Figure 8 shows the result with an extra $20 \mu \mathrm{m}$ movement in the optical path difference obtained by adding such a ramp. As in Fig. 5, these diagrams assume that all phototransistors are central.

The two-dimensional plots shown in Fig. 8 can be used to obtain an estimate of the correct alignment point for each detector using a method that finds the maximum point in the envelope of the two-dimensional surfaces. These estimates,
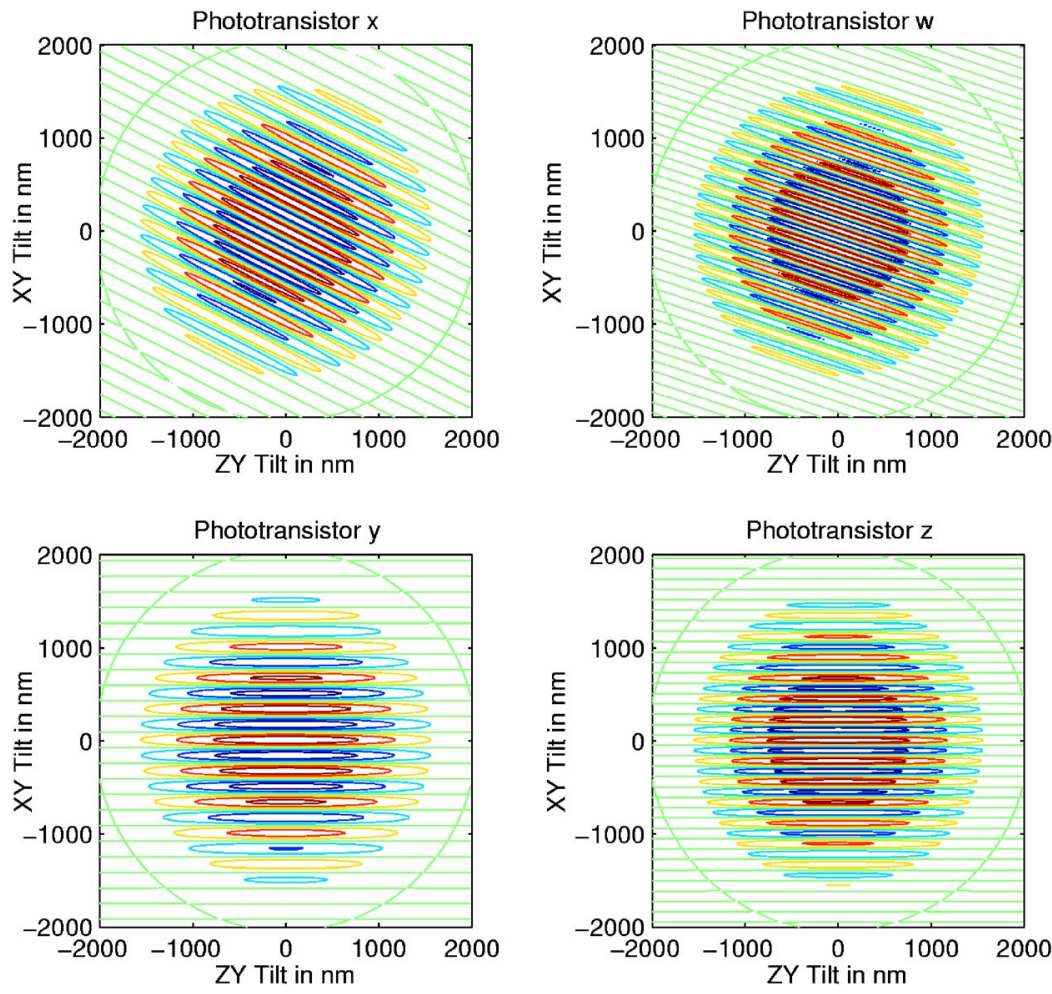

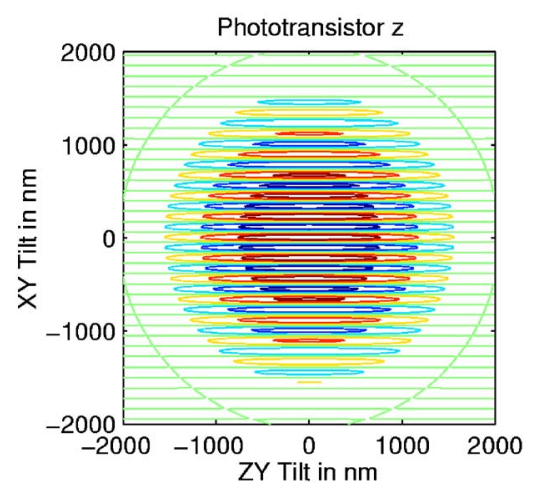

FIG. 8. Contours of all the expected phototransistor signals for different tilts and all the actuators moving. 


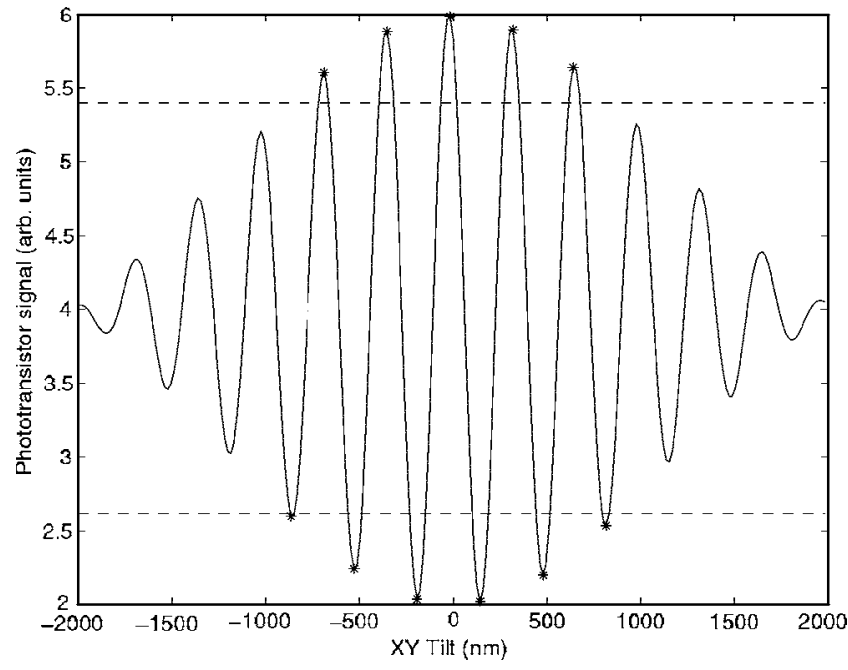

FIG. 9. Typical phototransistor signal for a scan in the $X Y$ tilt axis.

together with the geometry of the phototransistors and the main NIR detector, can be used to obtain four estimates of the correct alignment point.

A number of methods can be used to find the maximum points from the four phototransistor signals. In one method, the tilts for minimum attenuation can be found by fitting a quadratic surface to the peaks of the phototransistor signals, in the individual scans. Figure 9 shows a typical expected phototransistor signal for a scan in the $X Y$ tilt direction keeping the $Z Y$ tilt constant. This signal is a vertical cut-through of the phototransistor $x$ signal in Fig. 8. An algorithm was written to find the maximum and minimum peaks at this signal (plotted with asterisks in Fig. 9), within a threshold (dashed lines). To avoid local maxima/minima, it only finds one maximum between adjacent minima, and one minimum between adjacent maxima. So if there are two or more peaks above the threshold but not separated by minima below the negative threshold, only the highest of these peaks will be returned. At the peaks on this curve the cosine term in the phototransistor output will be unity. Therefore, a surface fitted to the peaks will approximate $b K(\alpha)$, where $b$ is a constant depending on the phototransistor gain, and $K(\alpha)$ is the attenuation due to tilt angle $\alpha$. So the peaks give points on the attenuation surface which can be approximated by a twodimensional quadratic function. Least squares fitting can be used to fit a quadratic to all the peaks which are sufficiently large. On our instrument we used all the peaks where the gain $K$ is at least 0.7 . Fitting a two-dimensional quadratic at both maxima and minima, it is possible to find by interpolation the points of minimum attenuation for each phototransistor. These points indicate the tilts required to align the mirrors. Any individual phototransistor could be used, but a better estimate is obtained by using all four phototransistors.

It is also possible to find the overall optimum by successive searches along orthogonal directions, e.g., first scan through a set of $X Y$ tilts while keeping the $Z Y$ tilt constant at $Z Y_{0}$. This gives us a "cut" through the surface as in Fig. 9. One-dimensional quadratics can be fitted to the maximum and minimum peaks in order to find the overall peak of the envelope containing the signal. This gives a best $X Y$ tilt $X Y_{1}$

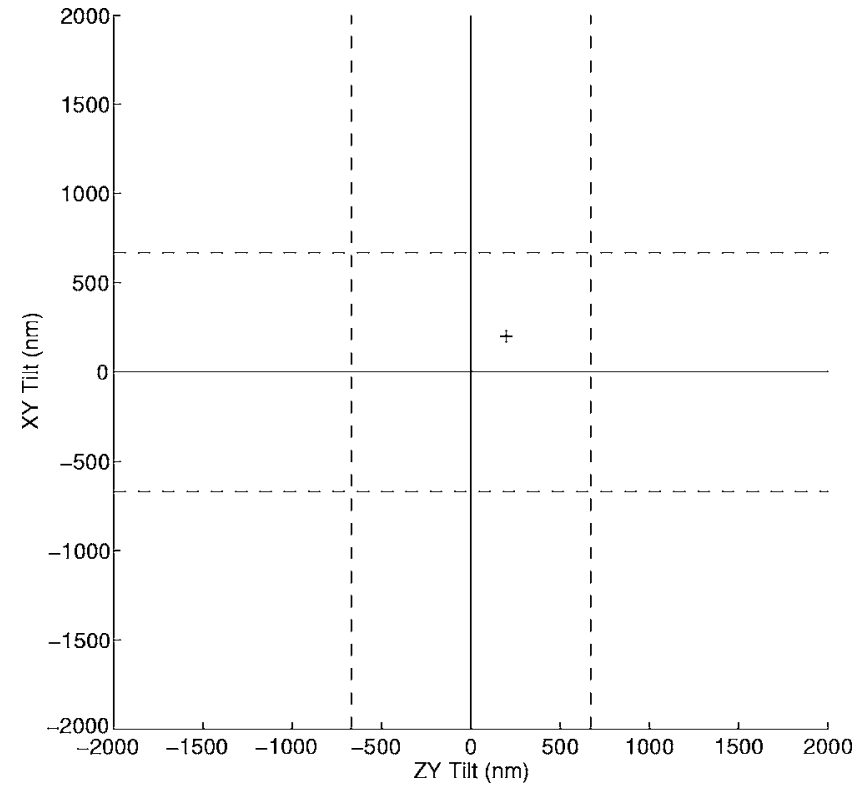

FIG. 10. Schematic diagram representing lines of tilt angles for which signals from different pairs of phototransistors are in phase.

for the $Z Y$ tilt $Z Y_{0}$. Next scan through a set of $Z Y$ tilts while keeping the $X Y$ tilt constant at $X Y_{1}$. This gives another cut through the surface as in Fig. 9. One-dimensional quadratics can be fitted again and give a best $Z Y$ tilt $Z Y_{1}$ for the original $X Y$ tilt $X Y_{1}$. The process is then repeated with the $Z Y$ tilt $Z Y_{1}$. This iteration converges fairly rapidly due to the overall surfaces in Fig. 8 having circular symmetry. A separate search has to be made for each phototransistor.

In practice the measured diffraction patterns are subject to noise, thus the earlier procedure is used to obtain estimates of the correct alignment angle for the mirror. With four such patterns we have four independent estimates of the correct alignment angle for the main $\mathrm{PbSe}$ photodetector. These estimates are based upon the amplitude properties of the twodimensional diffraction patterns. However, the diffraction patterns also contain important phase information, and so a second stage of the alignment process is added in which the relative phases of the detector signals are used to further improve the estimate of the amplitude maximum.

\section{Stage 2: Phase-based alignment}

When the mirrors are exactly aligned the optical path differences for the four phototransistors will be equal, and the phototransistor signals will be in phase. Therefore, the correct alignment point of the mirrors occurs at a pair of $X Y$ and $Z Y$ tilts which gives the same phases for each of the phototransistors $w, x, y$, and $z$. Given that the $x$ and $y$ phototransistors are spaced apart in a direction perpendicular to the direction of separation of the $y$ and $z$ phototransistors, any $Z Y$ tilt will not alter the relative phases of the $x$ and $y$ phototransistors. Therefore, there will be a number of $X Y$ tilt positions at which the outputs of the $x$ and $y$ phototransistors will be in phase regardless of the output of the $z$ phototransistor and equally there will be a number of values for $Z Y$ tilt at which the outputs of the $z$ and $y$ phototransistors will be in phase regardless of the output of the $x$ phototransistor. Figure 
10 shows three lines for which the $x$ and $y$ phototransistors signals are in phase (horizontal lines) and three for which the $z$ and $y$ phototransistors signals are in phase (vertical lines) with maximum amplitude. The cross represents tilt values corresponding to the initial alignment estimate produced by the first stage of the alignment process. The vertical solid line represents an $X Y$ tilt value for which the outputs of the $z$ and $y$ phototransistors are in phase regardless of $X Y$ tilt and the horizontal solid line represents a $Z Y$ tilt value for which the outputs of the $x$ and $y$ phototransistors are in phase regardless of $Z Y$ tilt. In this illustrative case, the intersection point of the two solid lines represents the true alignment point. Thus, in a practical situation, a second estimate of the true tilt position corresponding to alignment could be obtained from phase information and used to refine the estimate obtained from amplitude information.

In practice, there will be a number of lines parallel to the vertical solid line each of which represents a possible aligned position and a number of lines parallel to the horizontal solid line each of which corresponds to a possible aligned position (dashed lines in Fig. 10). The phase is periodic and so these lines repeat at tilt intervals of one wavelength $(670 \mathrm{~nm})$, so that the true maximum in the detector amplitude may be at any of the intersections of the zero-relative-phase lines. The tilt angles corresponding to these lines of the zero-relative phase are calculated and then used to improve the estimate of the alignment point obtained from the first stage.

At each of the peaks of the phototransistor signals found in the first stage, the phase difference will be zero or $2 \pi n$, where $n$ is an integer. Hence, by linear interpolation between the peaks we can find the phase of the signal at the particular phototransistor. This can be done for each point on the $X Y$ and $Z Y$ tilt planes shown in Fig. 8. The $x$ and $y$ phototransistors will have equal phase along a set of zero-relative-phase lines one wavelength apart in actuator positions, with constant $X Y$ tilt. The $z$ and $y$ phototransistors similarly will have equal phase along lines with constant $Z Y$ tilt. There should be a grid of points where these lines intersect, which are one wavelength apart in tilt angle in each direction, and where the phases of all four phototransistor signals are equal. The theoretical lines of equal phase can be calculated from the amplitude plots of Fig. 8 using linear interpolation and are presented in Fig. 11 for a typical simulation.

The intersection points of the lines in Fig. 11 correspond to points at which all detectors have the same phase. One of these intersection points should correspond to the zero phase point at which the amplitude is a maximum. In practice, however, this will not generally be the case due to inaccuracy in the estimation of the position of maximum amplitude. In addition, the lines in Fig. 11 are not straight as the calculation method used is necessarily approximate. Therefore, the accuracy of the second stage process is increased by finding the grid of points where the estimated phases of the phototransistors are closest. One of the ways of finding these points of the "closest phase" is to find the sum of the magnitudes of the phase differences between the phototransistors. Figure 12 shows the contours of the surface representing the sum of the magnitudes (again this picture is approximate due to the calculation method). The local minima are shown by

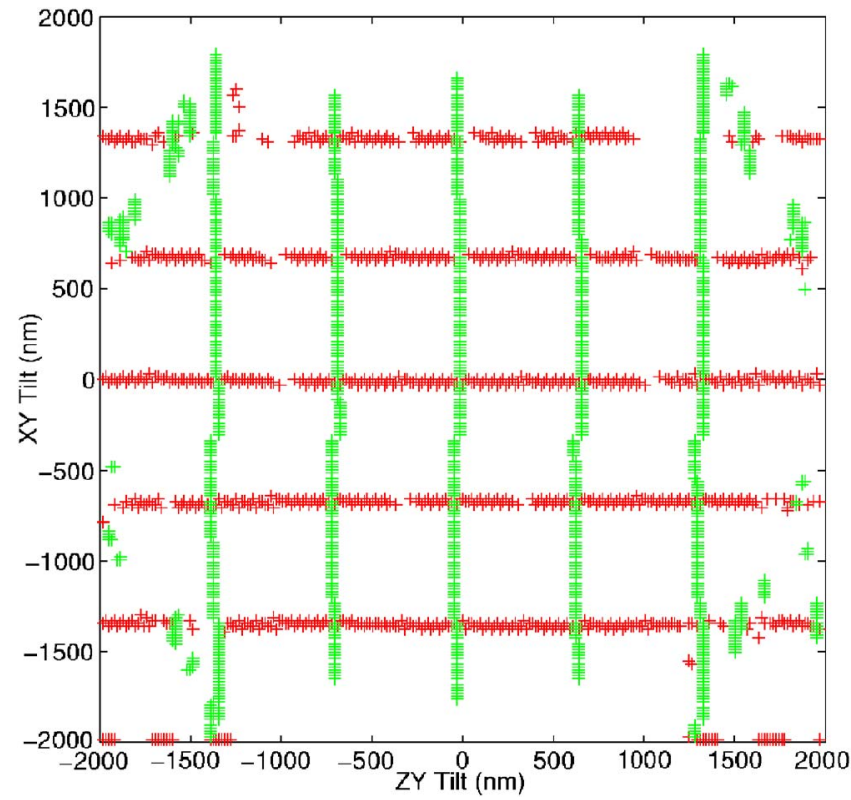

FIG. 11. (Color online) Mirror positions at which the phase is constant between the outputs of different pairs of phototransistors.

crosses. These crosses correspond to estimates of the tilt positions at which the lines of equal phototransistor phases cross, and all the phototransistors phases are equal. The vertical and horizontal solid lines are one wavelength apart and have been drawn in order to divide the area into squares. The point of the closest phase in each square is then plotted to form the grid pattern of Fig. 13.

One of these grid points in Fig. 13 corresponds to the mirrors being aligned with all four optical path differences being equal. The grid point nearest to the estimate obtained in the first stage is then selected as the new alignment point. However, this point (labeled 1 in Fig. 13) is an estimate, and the points around it (labeled 2-9 in Fig. 13) could also be accepted as possible estimates of the true alignment position. These points are tested in the third stage of the alignment algorithm to check the accuracy of the alignment and test for the best alignment point during the operation of the instrument.

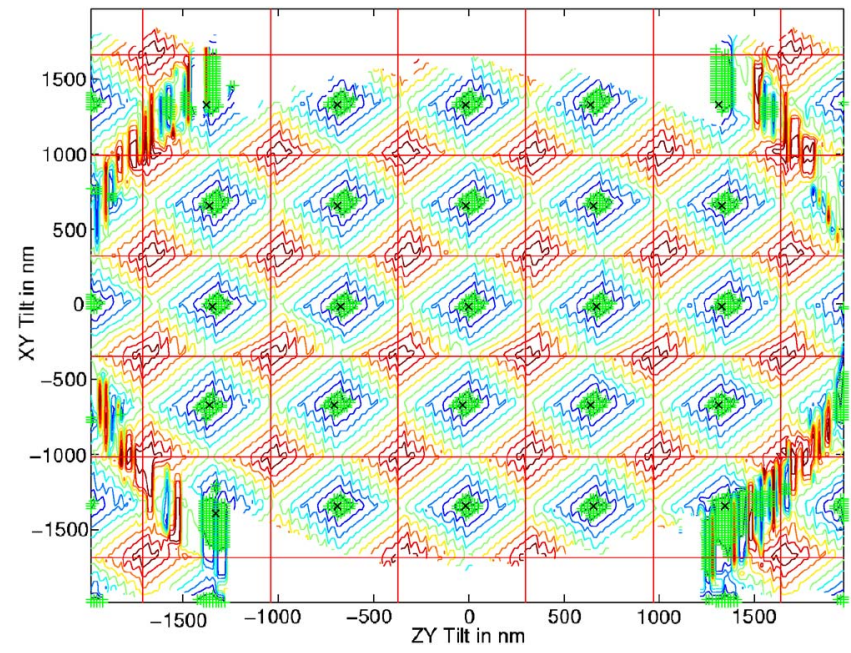

FIG. 12. (Color online) Contours of the magnitudes of phase differences. 


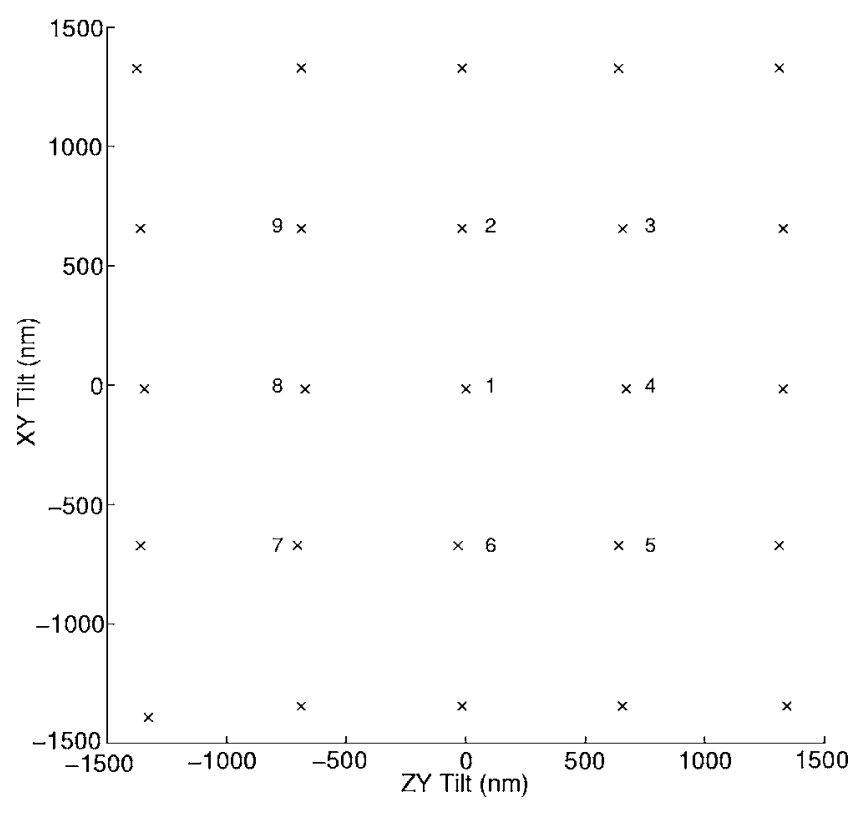

FIG. 13. Grid pattern of points at which the phase difference is minimum

\section{Stage 3: Dynamic alignment}

During normal operation, the active mirror is moved by applying the same displacement to all actuators attached to it. The normal sequence of operation is that the mirrors are initially aligned using the methods described in the first and second stages. This gives the set of actuators voltages that are required to place the interferometer's mirrors in alignment. A separate control scheme is then used during the scanning process. Its function is to keep the mirrors accurately aligned in real time, as the interferometer is scanning and also while it is idling between scans. This is known as dynamic alignment, and several such systems have been described in the literature. ${ }^{9-12}$ As described later, our system follows broadly similar principles but with an added capability to realign in the case of dynamic shock.

Our dynamic alignment algorithm ${ }^{13}$ uses the laser phototransistor signals $S_{x}, S_{y}$, and $S_{z}$, to control the active mirror's tilts during a scan. The laser source is monochromatic and therefore these signals are a set of sine waves. The dynamic control system measures the relative phases of the phototransistors sine waves and adjusts the actuators positions to maintain the relative phases at zero. This is done using a "position observer" attached to the outputs of the phototransistors and using the observer's outputs to drive the mirror alignment controllers. In this process, the laser phototransistor fringes are used for tracking the displacement of the mirrors using a form of the uniform time sampling method mentioned previously.

If the alignment algorithm worked correctly, then the zero phase point corresponds to the zero relative phase point marked as 1 in Fig. 13, and hence the zero phase point which gives the maximum amplitude signal at the main detector. However, the surrounding points 2-9 are also possible points for the correct alignment position. To test if these are better estimates than the one calculated from stages 1 and 2, a set of nine scans are collected using each one of the points as the initial alignment position. During this process, dynamic con- trol is used and the system monitors and compares the phototransistors signals amplitudes. At the end of these "test" scans the actuators voltages are adjusted so that the point of maximum signal is used as the mirrors alignment position during the interferogram collection process.

As noted earlier, the PZT actuators used to move the mirrors have a very fast response time-this means that their dynamics can be ignored in the overall control scheme as follows: a small delay is incorporated in the control software between piezoactuation and sampling the phototransistor outputs. This delay is selected to be just large enough to allow the actuators to have settled to their new steady state position. In this way the transducer dynamics do not influence the overall control process and the whole control scheme can be implemented free of dynamic consideration. This is possible because the initial alignment processwhich is the core of our method-is not time critical in a real-time control sense. In our current implementation, and with the actuator settling time delays included, the entire initialization takes less than $1 \mathrm{~min}$, a figure thought acceptable during the instrument "start-up" phase.

\section{B. Algorithm integration}

Initial and dynamic alignment algorithms are integrated into one system, which is fully automatic and capable of error detection and self-recovery.

After system's "power-up," stages 1 and 2 of the alignment algorithm are performed to calculate a good estimate of the alignment position of the mirror. Then, the scanning process and stage 3 follow, and the active mirror starts moving backwards and forwards applying the appropriate voltages to the PZTs. Stage 3 of the alignment is completed after nine scans and the correct alignment position is updated if necessary. During the scanning and interferogram collection process, the control system maintains alignment of the moving mirror by keeping the signal of the four phototransistors around the main detector in phase. At the end of each scan an error checking routine is executed. Its function is to check the phototransistors signals amplitude as well as the control system's performance and decide whether a scan should be stored or be discarded. This decision depends on two conditions which need to be met:

(1) each phototransistor's signal must be greater than a threshold and

(2) the sum of $X Y$ and $Z Y$ tilt errors must be less than or equal to the maximum allowed error $(\approx \lambda / 4)$.

If both of the conditions are met then the interferogram/ spectrum is a valid one and is displayed on screen. If one of the conditions is false, the current scan is discarded and the process is repeated. If excessive errors destabilized the control algorithms, e.g., due to excessive external disturbances, the system is reset and the instrument is aligned again from the beginning (stage 1). When the desired number of scans has been reached, the data collection process is terminated. These steps are shown graphically in Fig. 14 which describes the integration of the active alignment and the recalibration process after error checking. 


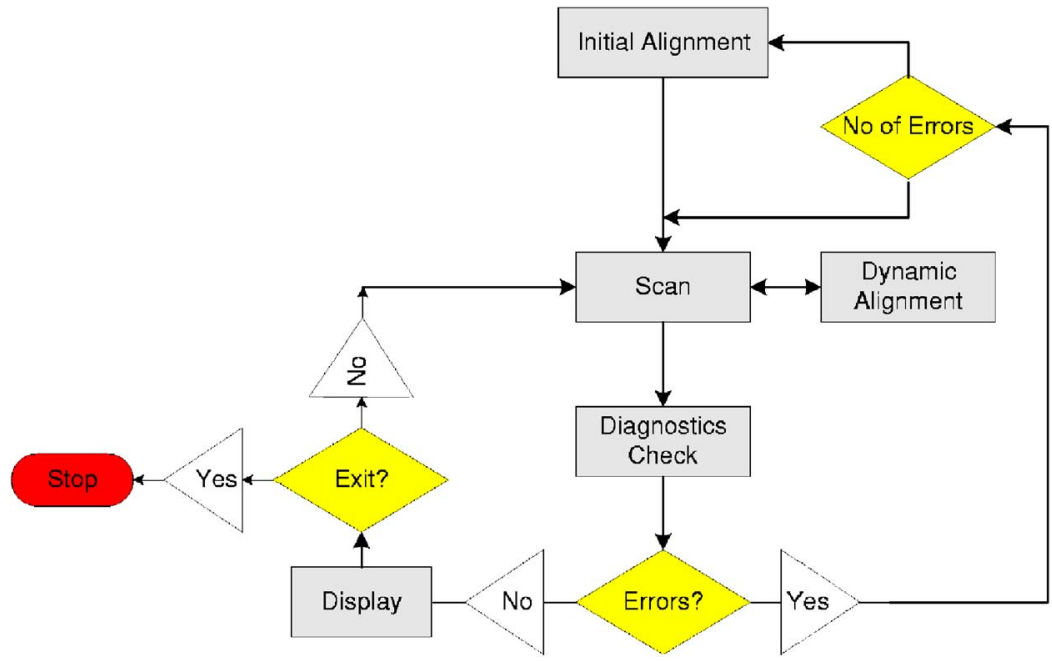

FIG. 14. (Color online) Diagram showing the integration of initial and dynamic alignment. Diagnostics test is performed at the end of the scan process.

\section{Validation}

The description of the active alignment method was illustrated in Sec. III A using simulated diffraction data. We now validate the method by showing representative results obtained from the experimental system described in Sec. II. As the method uses only monochromatic signals from the laser phototransistors, during the alignment process the white light was off. The execution time was $48 \mathrm{~s}$. Figure 15 shows the phototransistors signals of two-dimensional images for 256 scans with 256 points each. For better accuracy the number of scans or samples per scan could be increased. That would slow down the process though. To obtain these images, the mirror was tilted by $2 \mathrm{~V}$ (from -1 to $1 \mathrm{~V}$ ) in both axes, $Y X$ and $Y Z$. A ramp change of $4 \mathrm{~V}$ was also added to all the actuators simultaneously. The phototransistor signals are similar to the simulated signals in Fig. 8. There are some expected differences mainly due to internal reflection of the optical components of the interferometer, which are more visible in phototransistor $y$ 's signal. The signal from phototransistor $x$ is shown at larger scale separately in Fig. 16. The algorithm uses the two-dimensional images of Fig. 15 to calculate the phototransistors alignment positions and then the first estimate of the NIR detector's position based on the amplitude of the phototransistors signals (stage 1). For this specific experiment the estimated positions were

$$
\begin{aligned}
& \left(V_{Z Y}^{w}, V_{X Y}^{w}\right)=(0.0182,0.2895), \\
& \left(V_{Z Y}^{x}, V_{X Y}^{x}\right)=(0.1100,0.3480),
\end{aligned}
$$
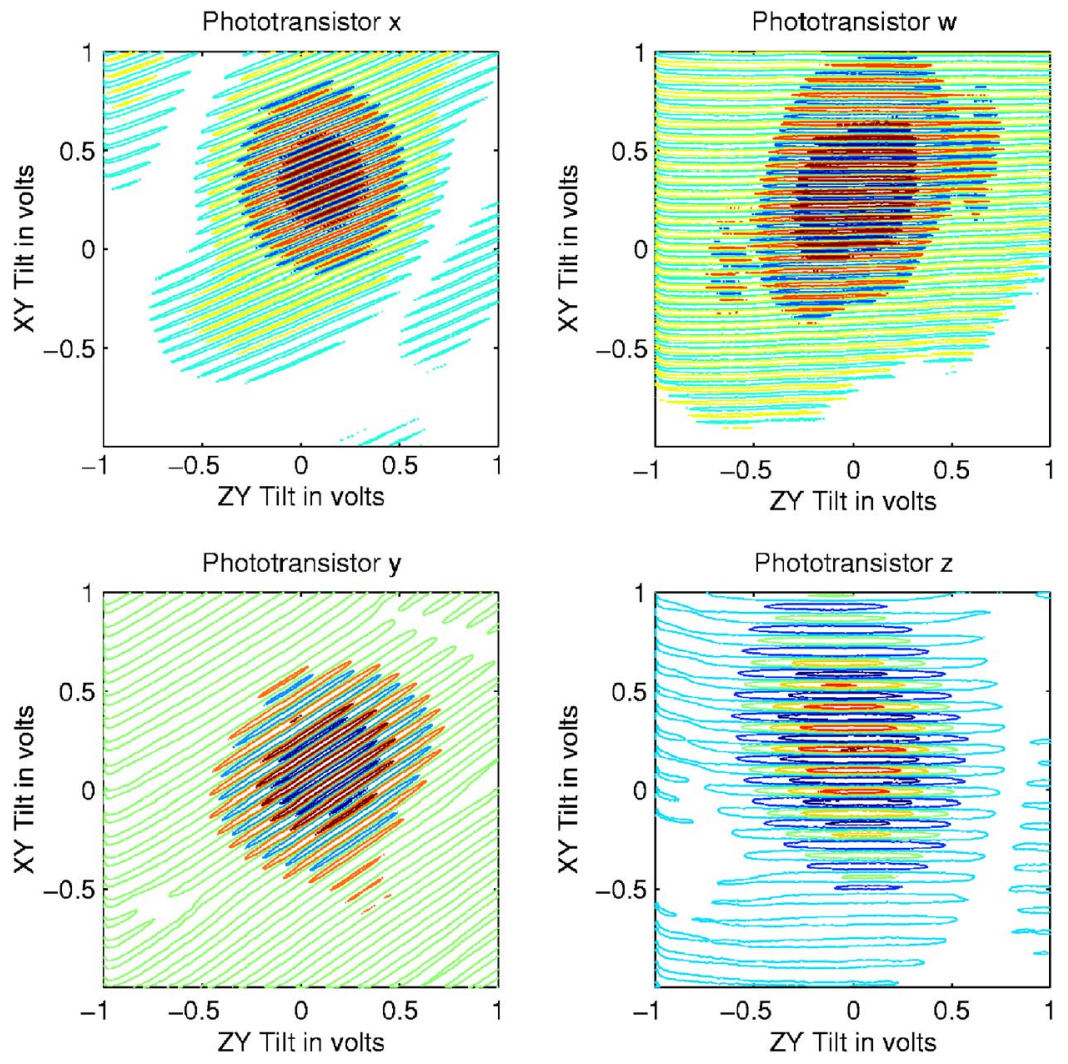

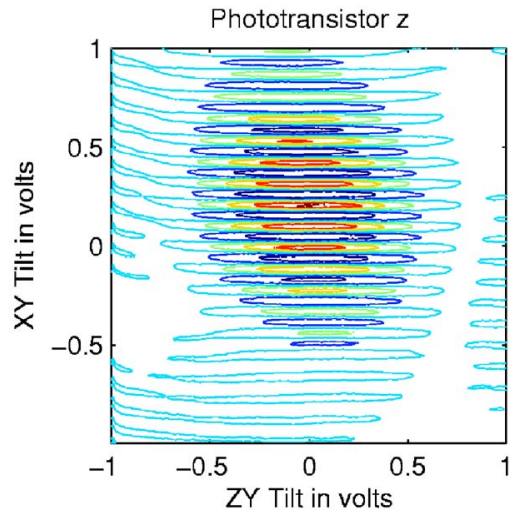

FIG. 15. (Color online) Contours of phototransistor experimental signals for different tilts and all the actuators moving. 


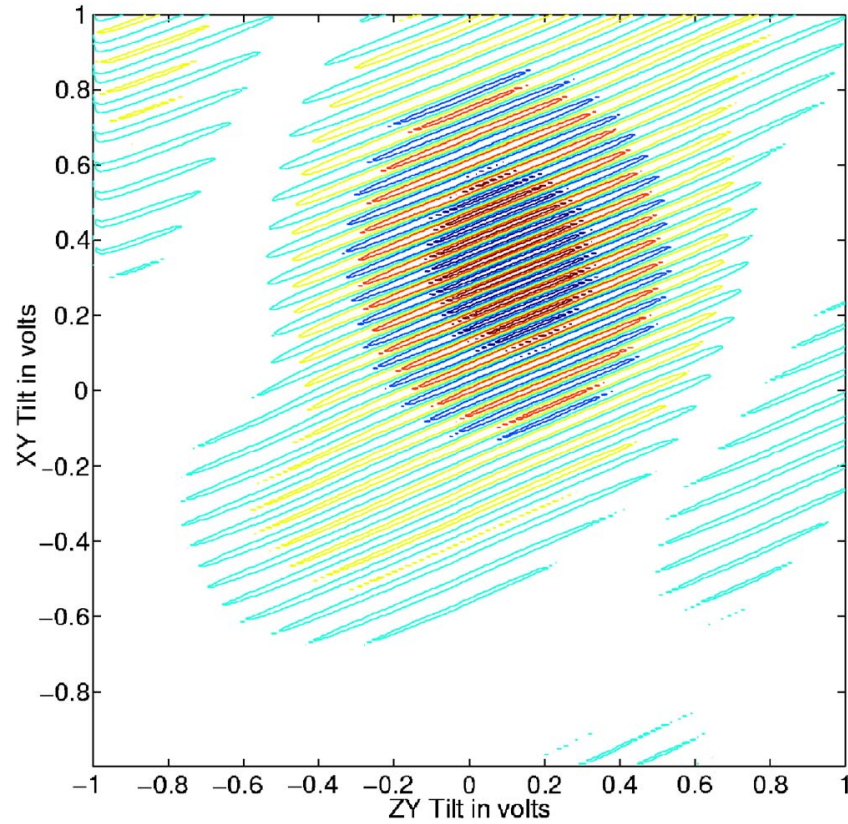

FIG. 16. (Color online) Contour of phototransistor $x$ experimental signal for different tilts and all the actuators moving.

$$
\begin{aligned}
& \left(V_{Z Y}^{y}, V_{X Y}^{y}\right)=(0.1272,0.1116), \\
& \left(V_{Z Y}^{z}, V_{X Y}^{z}\right)=(-0.0136,0.219) .
\end{aligned}
$$

The positions are expressed in volts. The first estimated position of the NIR detector is then calculated by averaging the positions of the phototransistors as follows:

$$
\begin{aligned}
& V_{Z Y}^{d e}=\frac{V_{Z Y}^{w}+V_{Z Y}^{x}+V_{Z Y}^{y}+V_{Z Y}^{z}}{4}, \\
& V_{X Y}^{d e}=\frac{V_{X Y}^{w}+V_{X Y}^{x}+V_{X Y}^{y}+V_{X Y}^{z}}{4} .
\end{aligned}
$$

Equations (7) and (8) give

$$
\left(V_{Z Y}^{d e}, V_{X Y}^{d e}\right)=(0.0600,0.2442),
$$

which means that the interferometer is aligned if the $X$ actuator gets a voltage of $0.2442 \mathrm{~V}$ and the $Z$ actuator $0.06 \mathrm{~V}$ while the voltage at the $Y$ actuator is kept constant.

The lines of equal phase are then calculated from the amplitude plots of Fig. 15 using linear interpolation and are presented in Fig. 17. As in Fig. 11, which is the corresponding representation with simulated phototransistors signals, the lines of crosses are not straight as the prototype is not perfect and the calculation method used is necessarily approximate. The estimated positions of the phototransistors

TABLE I. Center wavelengths and bandwidths of interference filters as specified by the manufacturer. (FWHM: full width at half maximum.)

\begin{tabular}{lcc}
\hline \hline Filter No. & Center wavelength $(\mathrm{nm})$ & Bandwidth FWHM (nm) \\
\hline 1 & $1200 \pm 3.5$ & $11.2 \pm 5.0$ \\
2 & $1400 \pm 3.5$ & $13.6 \pm 5.0$ \\
3 & $1600 \pm 3.5$ & $15.7 \pm 5.0$ \\
4 & $1800 \pm 3.5$ & $17.0 \pm 5.0$ \\
5 & $1940 \pm 3.5$ & $18.7 \pm 5.0$ \\
\hline \hline
\end{tabular}

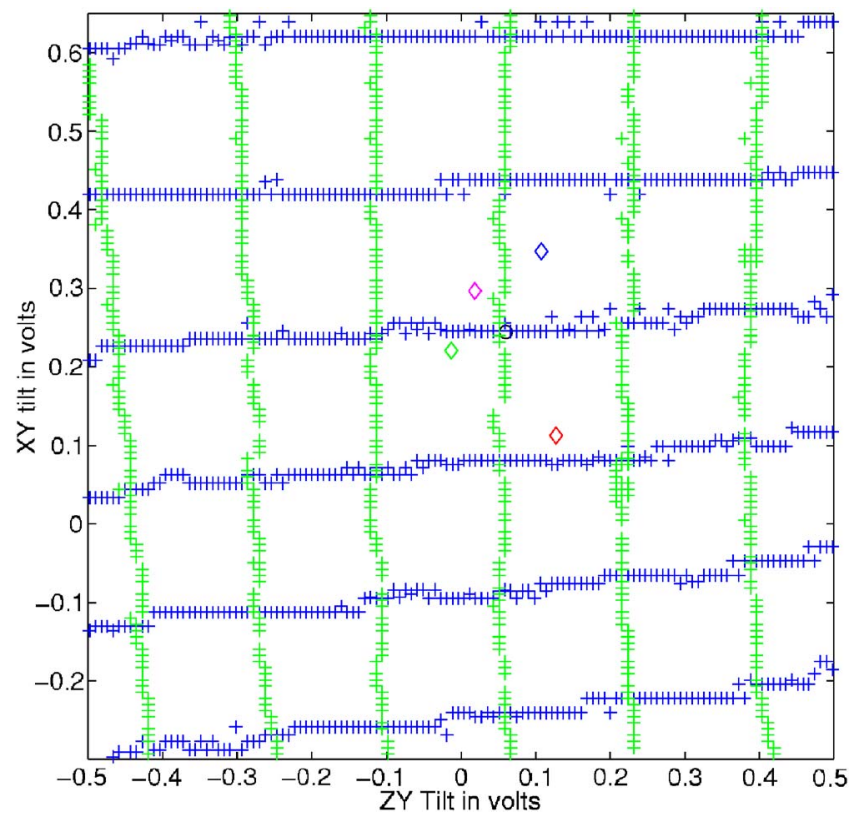

FIG. 17. (Color online) Mirror positions at which the phase is constant as between the outputs of different pairs of phototransistors (practical case).

and the detector from the first stage of the initial alignment method, have been also plotted. The top left diamond corresponds to phototransistor $w$, and then clockwise to phototransistors $x, y$, and $z$. The first estimate for the detector's position is shown with a circle.

The process continues with the calculation of the points of the closest phase by finding the sum of the magnitudes of the phase differences between the phototransistors. Figure 18 shows the contours of the surface representing the sum of the magnitudes for the experimental case, in a similar way to Fig. 12 where simulated signals were used. Again, the local minima are shown by crosses. These crosses that correspond

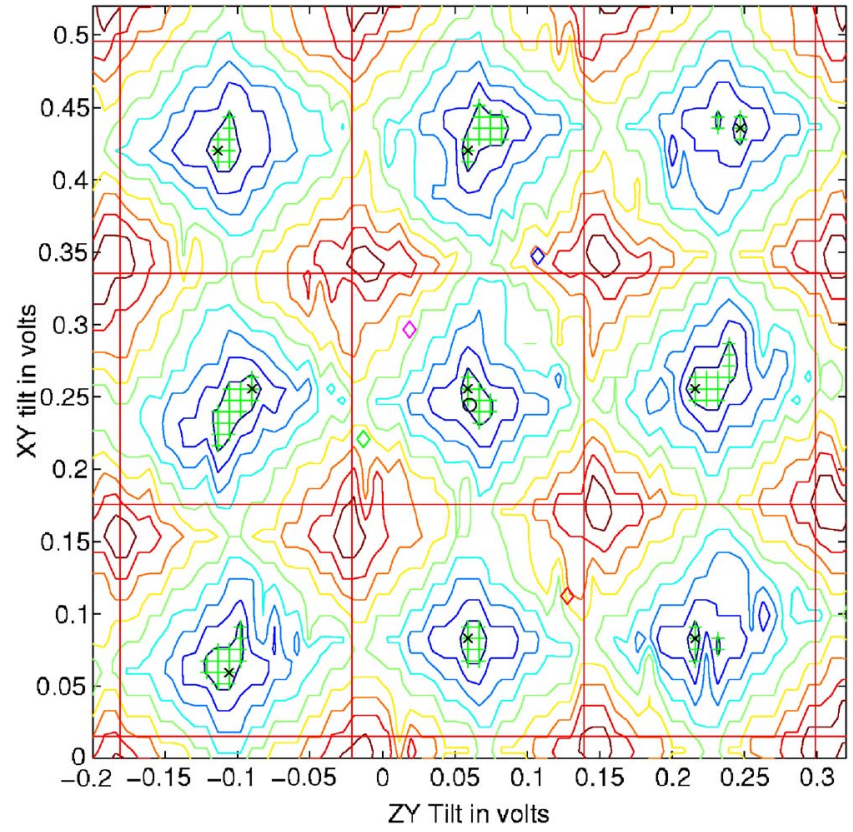

FIG. 18. (Color online) Contours of the magnitudes of phase differences (practical case). 


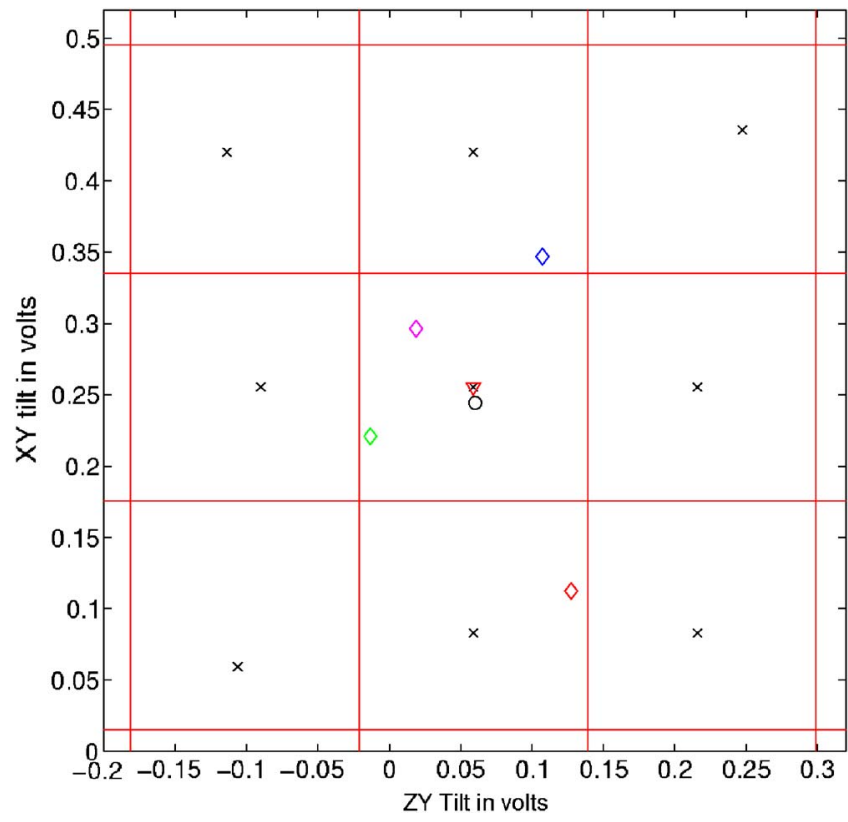

FIG. 19. (Color online) Estimated phototransistor positions plotted together with points at which the phase difference is minimum.

to estimates of the tilt positions at which all the phototransistors phases are equal, are then plotted to form the grid pattern of Fig. 19, where the positions of the phototransistors and the detector can be also seen. Both Figs. 18 and 19 have been zoomed around the area of the estimated alignment position from stage 1 . The algorithm then picks the grid point nearest to the estimate obtained in the first stage as the best estimate of the true alignment point, marked with a triangle. The tilt voltages for this alignment point as calculated for this experiment, were

$$
\left(V_{Z Y}^{d e}, V_{X Y}^{d e}\right)=(0.0588,0.2555) .
$$

This is the information that is used later as the initial alignment point for stage 3 and is tested together with the eight points around it during operation of the instrument using dynamic control. For this particular case, the point chosen from stages 1 and 2 gave the larger phototransistors signals and it was used as the correct alignment position.

\section{RESULTS AND DISCUSSION}

\section{A. Typical Results}

In order to illustrate the spectral calibration of the instrument while operating with the active control system, a series of experiments were undertaken using narrow band interference filters. The particular bands chosen covered the region

TABLE II. Center wavelengths and bandwidths of the interference filters as determined from experimental data.

\begin{tabular}{lcc}
\hline \hline Filter No. & Center wavelength $(\mathrm{nm})$ & Bandwidth FWHM $(\mathrm{nm})$ \\
\hline 1 & 1203.5 & 56.51 \\
2 & 1401.7 & 75.42 \\
3 & 1595.6 & 99.4 \\
4 & 1797 & 127.3 \\
5 & 1933.1 & 150.81 \\
\hline \hline
\end{tabular}

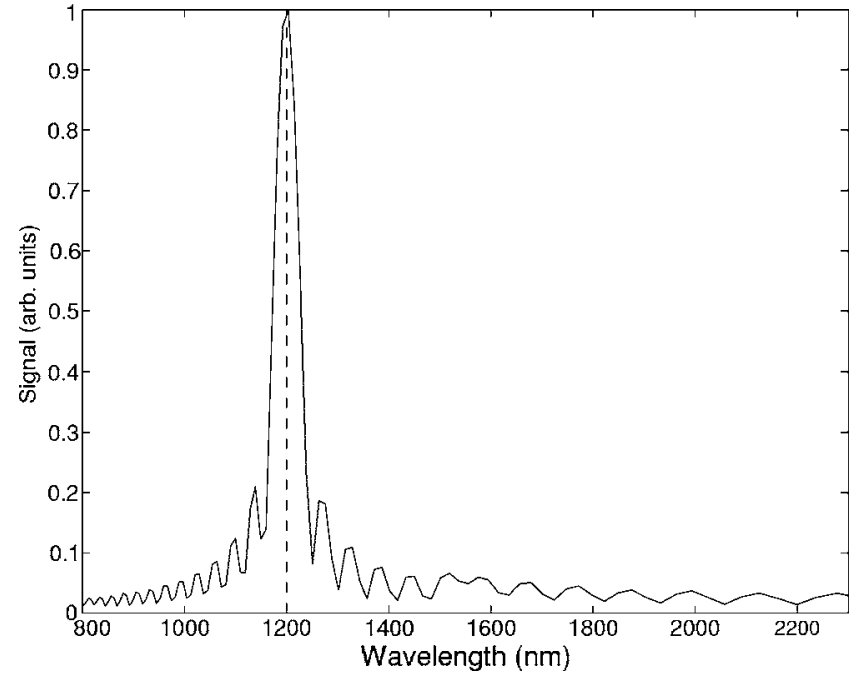

FIG. 20. Transmission spectra of bandpass filter 1 with center wavelength $1200 \mathrm{~nm}$.

of interest of the spectrum. The center wavelengths and bandwidths for each filter are listed in Table I as specified by the manufacturer (Ealing Catalog, Inc.).

The instrument was used in transmission mode for the calibrations, as shown in Fig. 2. Fifty interferograms were recorded for each filter. Fourier transformation of the interferograms followed by spectral averaging produced the results shown in Figs. 20-22 for filters 1, 2, and 4, respectively. The plots show that the center wavelengths were resolved correctly. The spread of each spectrum is the result of convolving the filter's response with the instrument function. Table II lists the center wavelengths and bandwidths of each filter as determined from the experimental data. A comparison of the experimental bandwidths to those stated by the manufacturer shows that the instrument function has a considerable effect on the shape of the spectra which was increased when moving to longer wavelengths. In particular, in the experimental setup described here the maximum mirror retardation was small (of the order of $30 \mathrm{~nm}$ ), hence, the

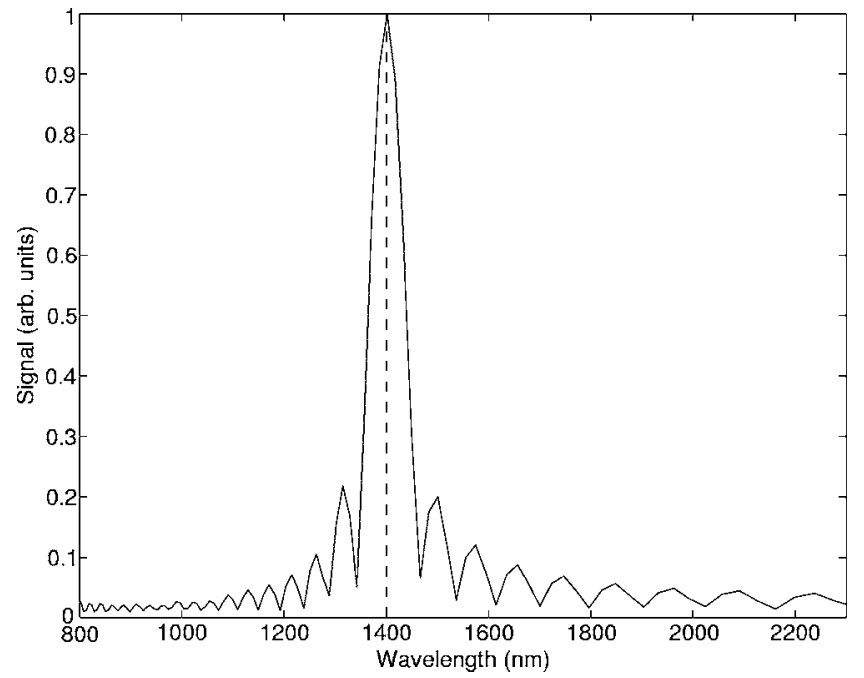

FIG. 21. Transmission spectra of bandpass filter 2 with center wavelength $1400 \mathrm{~nm}$. 


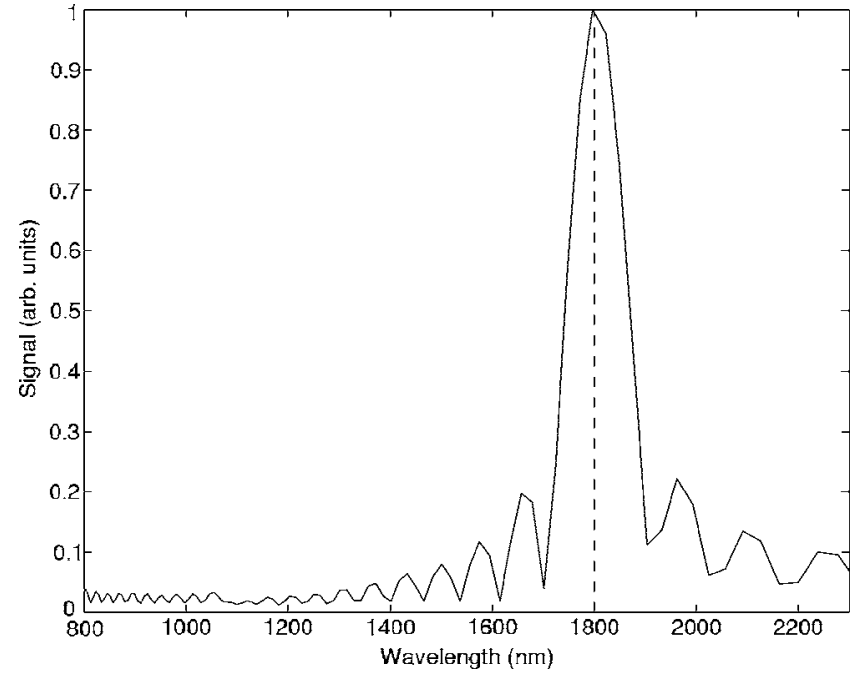

FIG. 22. Transmission spectra of bandpass filter 4 with center wavelength $1800 \mathrm{~nm}$.

relatively wide bandwidth of the measured filter responses.

In Figs. 20-22, the sidelobes of the spectra indicate the presence of spectral leakage. This phenomenon is another side effect of the convolution of the filter response with the instrument function. In order to address this problem, several windowing functions were applied to the interferograms. The values of the highest sidelobe and an estimate of the bandwidth using Bartlett, Hamming, Blackman, and Tukey functions, ${ }^{14}$ can be seen in Table III for the case of filter 1. Figures 23 and 24 show the original spectrum from filter 1 together with the normalized spectra obtained from interferograms windowed using Hamming and Tukey functions, respectively.

\section{B. Discussion}

The active alignment method described here provides several benefits. First, initial alignment may be automatically implemented, thereby removing the necessity for this task to be carried out by a skilled operator. Second, alignment may be continuously or frequently checked and maintained. This increases confidence in the integrity of the measurement and offers the possibility of an automatic warning if for any reason the alignment fails and cannot be automatically restored. This has obvious benefits in an situation where the instrument is used in any application where there are regulatory, safety, or other implications in the event of a loss of measurement accuracy. Third, it prevents a progressive deterioration in performance after initial alignment, and so has im-

TABLE III. Figures of merit for the four window functions applied to filter 1.

\begin{tabular}{lcc}
\hline \hline Window & Highest sidelobe $(\mathrm{dB})$ & Bandwidth $(\mathrm{nm})$ \\
\hline Bartlett & -13.93 & 83.60 \\
Hamming & -14.66 & 85.92 \\
Hann & -14.89 & 94.03 \\
Blackman & -14.8 & 108.28 \\
Tukey & -7.71 & 70.03 \\
\hline \hline
\end{tabular}

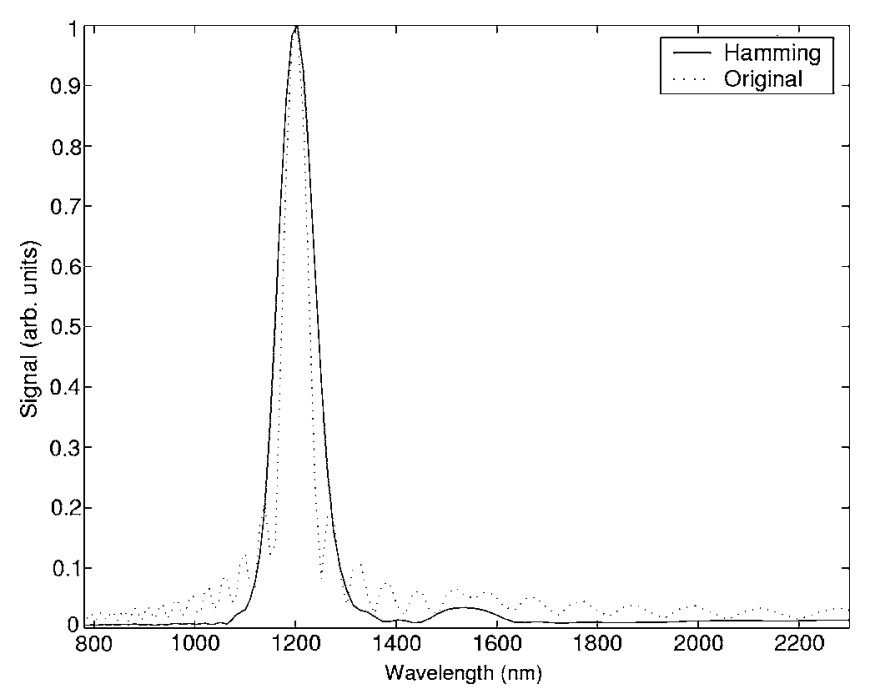

FIG. 23. Original spectrum and spectra obtained by windowing the interferograms of filter 1 with Hamming function. Original spectrum has been plotted with dotted line where windowed spectra with solid line.

plications for reducing the amount of routine service that may be required. Related to this is the possibility that the instrument can be designed and engineered to poorer tolerances, especially with regard to mechanical stability, thereby reducing costs. The ability of the instrument to maintain alignment during operation offers the potential for using the instrument in adverse conditions in which prior art instruments would not be capable of reliable operation for extended periods of time. This is especially important when it is desired to extend the use of measurement currently effected as a laboratory check onto a production line for example. The economic benefits of making a measurement continuously on a production line are frequently much greater than making the same measurement intermittently on samples taken from that line. Benefits typically include the saving of energy, the maintenance of high product quality and the reduction of the quantity of waste or defective product.

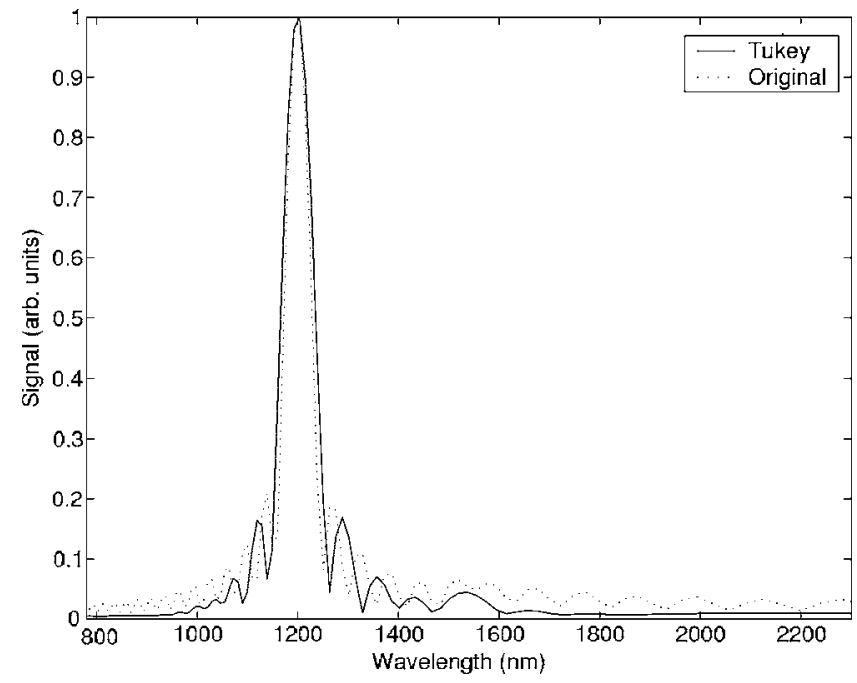

FIG. 24. Original spectrum and spectra obtained by windowing the interferograms of filter 1 with Tukey function. Original spectrum has been plotted with dotted line where windowed spectra with solid line. 
The results shown in the previous section are representative and active control alignment has been shown to function consistently and well in a range of embodiments. In particular the experimental system described in Sec. II was based upon system in which the alignment and retardation were combined in one mirror. A more useful embodiment has the alignment operation in one mirror and the retardation in the other mirror. This allows the larger mirror retardation required in a practical instrument, and can be achieved with only small software modifications.

Dynamic control of alignment during mirror retardation is well known and for that reason we have concentrated on the initial alignment part of the active control method. Our only additions to dynamic control have been in the use of "position observers" and a form of uniform time sampling, and these are described in detail elsewhere. ${ }^{13}$ Likewise, we have only mentioned the use of active control in instrument diagnostics - this is because its implementation is an obvious consequence of the alignment measurement method.

\section{DISCUSSION}

A control system has been described for the automatic alignment of two-beam interferometers. The basic aim is to automate the initial alignment of an instrument, as well as the realignment after shock or severe environmental change. Our motivation for this work has been the need to use the results of FT-NIR at the point of measurement, that is to say at-line or preferably on-line. The growing demands of Process Analytical Technology place a responsibility on instrument designers to make instruments suitable to this purpose.
It is our claim that the active control system described here is a useful core technology for on-line FT-NIR instruments.

\section{ACKNOWLEDGMENTS}

The financial support of this work by the Engineering and Physical Sciences Research Council, as well as the Manchester Technology Fund, are gratefully acknowledged. Credit is also due to Ralph J. Houston and Simon M. Christie for partial construction of the earlier version of the instrument.

${ }^{1}$ P. Griffiths and J. de Haseth, Fourier Transform Infrared Spectrometry (Wiley, New York, 1986).

${ }^{2}$ P. Wellstead and J. Edmunds, "Interferometer Optical Element Alignment," Patent No. PCT/GB2003/003664, 2003.

${ }^{3}$ C. Williams, Appl. Opt. 5, 1084 (1966).

${ }^{4}$ D. Cohen, Appl. Opt. 36, 4034 (1997).

${ }^{5}$ F. Stremler, Introduction to Communication Systems (Addison-Wesley, Reading, MA, 1990).

${ }^{6}$ J. Connes, Rev. Opt., Theor. Instrum. 40, 45 (1961).

${ }^{7}$ J. Workman, Applied Spectroscopy: A Compact Reference for Practitioners (Academic, San Diego, CA, 1998), Chap.: Optical Spectrometers.

${ }^{8}$ J. Brasunas and G. Cushman, Appl. Opt. 36, 2206 (1997).

${ }^{9}$ Z. Lu, R. Koehler, W. Gault, and F. Liang, J. Phys. E 21, 71 (1988).

${ }^{10}$ E. Morrison, B. Meers, D. Robertson, and W. Henry, Appl. Opt. 33, 5041 (1994).

${ }^{11}$ F. Barone, L. Di Fiore, L. Milano, G. Russo, and S. Solimeno, IEEE Trans. Nucl. Sci. 39, 232 (1992).

${ }^{12}$ K. Goldberg, P. Naulleau, and J. Bokor, Appl. Opt. 41, 4477 (2002).

${ }^{13}$ D. Kalamatianos, J. Edmunds, P. Wellstead, R. Houston, P. Liatsis, S. Christie, R. Dewhurst, and M. Thorniley, Proceedings of the IEEE International Conference on Virtual Environments, Human-Computer Interfaces and Measurement Systems (Boston, MA, 2004), pp. 120-124.

${ }^{14}$ F. Harris, Proc. IEEE 66, 51 (1978). 